\title{
Appetitive Feeding Behavior of Aplysia: Behavioral and Neural Analysis of Directed Head Turning
}

\author{
Thomas Teyke,, Klaudiusz R. Weiss, ${ }^{b}$ and Irving Kupfermann \\ Center for Neurobiology and Behavior, College of Physicians and Surgeons of Columbia University, New York, \\ New York 10032
}

The appetitive phase of feeding behavior in Aplysia consists of a behavioral sequence in which the quiescent animal starts to locomote and then assumes a characteristic feeding posture. In this position, head-turning responses can be elicited by a localized food stimulus (seaweed) delivered to the lips or tentacles.

In response to brief (open loop) stimulation with seaweed, the animal turns toward the stimulus but greatly overshoots the target. However, the angular velocity and the final turning angle are a function of the eccentricity of the stimulus, progressively increasing with greater eccentricities. In a foodaroused animal, a brief tactile stimulus evokes turning and biting responses similar to those triggered by seaweed, which provides both tactile and chemical stimulation. Upon repeated tactile stimulation, however, the response magnitude decrements rapidly, whereas the magnitude remains high when turning responses are repeatedly elicited by food stimuli. A purely chemical stimulus sometimes can elicit a turning response, but chemical stimuli alone are much less efficacious than tactile stimuli alone. When the stimulus is maintained in a stationary position (closed loop), the animal turns until its mouth is oriented over the food. A turning response to a lateral stimulus can be reduced by an immediately following medlal stimulus. To explain the above findings, we propose a form of response substitution, in which the response to the first, lateral stimulus is substituted by a weaker response to a more medial stimulus. No turning response is evoked when the animal is stimulated while performing spontaneous or evoked bites, though biting per se does not interrupt ongoing turning movements. In animals with lesions of the cerebral-buccal connectives, a food stimulus on the mouth is also followed by a reduction of the capacity of stimuli to elicit turning responses. In these lesioned animals, the food stimulus appears to elicit a bite command, though the biting behavior itself does not occur. Thus, it appears that the bite-related gating of stimuli is of cerebral origin, rather than due to the generation of the buccal motor program.

\footnotetext{
Received Apr. 9, 1990; revised July 20, 1990; accepted Aug. 20, 1990.

This work was supported by U.S. Public Health Service Grants MH 35564 and GM 320099 to I.K and DF́G Grant Te 138/1-1 to T.T.

Correspondence should be addressed to Dr. Irving Kupfermann, Center for Neurobiology and Behavior, College of Physicians and Surgeons of Columbia University, 722 West 168th Street, Research Annex, New York, NY 10032.

a Present address: Institut für Zoologie (III) Biophysik, Johannes GutenbergUniversität, Saarstrassee 21, 6500 Mainz, FRG.

${ }^{\circ}$ Present address: Department of Physiology and Biophysics, Mt. Sinai School of Medicine, New York, NY 10029.

Copyright (C) 1990 Society for Neuroscience $0270-6474 / 90 / 123922-13 \$ 03.00 / 0$
}

The force necessary to power the turning movements was calculated from the trajectories of the movements. The results indicate that a power phase during the first half of the duration of the total movement is sufficient to generate a turn. The power phase can be followed by a brief gliding phase, and finally the movement appears to be actively terminated. The timing and power phases inferred from the results of the calculations were qualitatively tested by comparing the calculated torque to activity recorded from neck nerves and the neck muscles in free-moving animals. Increased unit activity in the nerves and the neck muscles ipsilateral to the turning direction occurred mostly at the beginning of the movement, supporting the notion of a brief power phase. Nerves contralateral to the movement exhibited an increase in activity just before termination of the movement. Turning movements to all angles appear to be generated by a concerted action of several motor units innervating the neck via different pleural/pedal nerves.

Feeding behavior of Aplysia provides an advantageous model system for the study of complex behaviors and motivational states (see Kupfermann, 1974a). Previous studies by Kupfermann (1974b) have shown that feeding in Aplysia, similar to motivated behaviors in higher animals, can be separated into appetitive and consummatory phases (see Craig, 1918). Appetitive feeding responses in Aplysia can be further divided into 2 phases. In phase 1 , in response to food, a hitherto quiescent animal starts to locomote and eventually assumes a characteristic feeding posture, in which the posterior portion of the foot is attached to the substrate, and the anterior part of the body (head, neck, and anterior foot) are lifted off the substrate (Frings and Frings, 1965). In addition, other behavioral responses in the animal, such as withdrawal responses, are modified (Advokat, 1980). When the animal is oriented in the feeding posture, contact with potential food sources elicits a second phase of appetitive behavior; that is, the animal turns its anterior body in the direction of the stimulus. Stimulation on the mouth then triggers stereotypic biting responses, which are referred to as consummatory behaviors, because they consummate the behavioral sequence and attain the goal object.

Previous studies have examined behavioral and neural aspects of consummatory behaviors (Susswein et al., 1978; Weiss et al., 1978; Rosen et al., 1988; Cropper et al., 1990), and of the first phase of appetitive behaviors (Teyke et al., 1990). This paper explores the second phase of appetitive behavior, in which the animal orients its head in the direction of a contact food stimulus. Different stimulation protocols were employed in order to study the input-output function of the evoked turning 
response. For further analysis, we calculated the force generating the turning movement and compared the results to the pattern of the neuronal output recorded from nerves that innervate the neck muscles. The overall data suggest a novel model for the neural control of orienting responses, and the model provides testable hypotheses of motivational state in regulating appetitive behaviors.

\section{Materials and Methods}

Animals. Subjects were Aplysia californica (weighing 150-250 gm) obtained from Marinus, CA. The animals were kept in individual holding cages $(30 \times 20 \times 10 \mathrm{~cm})$ that were mounted within a 600 -liter tank and circulated with filtered, aerated, and cooled $\left(15^{\circ} \mathrm{C}\right)$ artificial seawater (Instant Ocean). Before being used in an experiment, the animals were first fed with dried seaweed (Laver), then deprived of food for at least $3 \mathrm{~d}$. They received no further food throughout the experiments.

Experimental procedure. All experiments were performed on animals that wcre first aroused with a piece of food that was touched to the rhinophores. The animals were further stimulated until they assumed a feeding posture in which the posterior part of the foot was attached to the wall, while the anterior part of the animal (including the foot, lips, and tentacles) was free from the substrate and in contact with the surface of the water. Figure 1 shows a diagrammatic top view of the animal in the final feeding posture. In this position, the anterior part of the animal is free to turn left or right along a horizontal plane, and in addition, it can arch downwards. Unless stimulated, the animal typically remained motionless, but occasionally performed relatively slow spontaneous turning movements. When the animal was stationary in a middle (centered) position, a seaweed stimulus was touched on different spots on the lips or tentacles. The stimulus consisted of a moistened 2$\mathrm{mm}^{2}$ piece of seaweed (Laver), which was applied with a fine, blunt forceps. The scawced stimulus provides tactilc as wcll as chemical stimulation. Application of the tip of a heat-sealed glass pasteur pipette provides a purely tactile stimulus. Animals were studied in either an open-loop or closed-loop condition. In the open-loop condition, the stimulus was applied briefly (approximately $0.5 \mathrm{sec}$, or for varying durations specified in the description of the experiment) at a given locus and then removed. In the closed-loop condition, the animal was touched with a piece of seaweed, which was kept in a fixed position. Thus, when the animal moved its head while it executed a turning movement, the seaweed moved across the lips, so that the animal received stimulation from different loci on its receptive surface. The seaweed was removed immediately before the animal moved to a position in which it could consume the food.

Figure 1 shows a typical response elicited under open-loop conditions and illustrates the method of measuring the turning angle. The animal was briefly stimulated with a piece of seaweed at a $10^{\circ}$ eccentric location (Fig. 1, vertical arrow), measured with respect to the center axis (dashed center line), with the mouth orifice serving as the $0^{\circ}$ reference point. After a short latency, the animal responded with a directed turning movement in the direction of the stimulated side. In the case depicted in Figure 1, the animal terminated its turning movement at a final turning angle of approximately $50^{\circ}$ (measured at the intersection of the center lines). Following that response, the animal moved slowly, and in several steps, back to the middle position. An overhead video camera was used to record the position of the animal on videotape, and the turning angle was measured from stopped frames, sampled every 250 msec, directly from the monitor. Because the body of the animal bends rather than swings around a pivotal point, a pivot point was defined for cach turning response by using the intersection of the initial and the final center lines (see Fig. 1). Cases in which the center line could not be tracked clearly, for example, because it became obscured by the closing lips, were not included in the analysis.

Except as otherwise indicated, the angular position of the stimulus in different trials was varied in an irregular order. Both the left and the right sides of the animal were stimulated, but because none of the animals showed a strong asymmetry in its response towards a particular side, the data for both directions were pooled. The minimal time interval between 2 consecutive stimulations was $30 \mathrm{sec}$. In a number of cases, because of spontaneous movements or spontaneously occurring bites, longer interstimulus intervals were used. To address specific questions, variations of the above protocol were employed and are indicated in the Results.

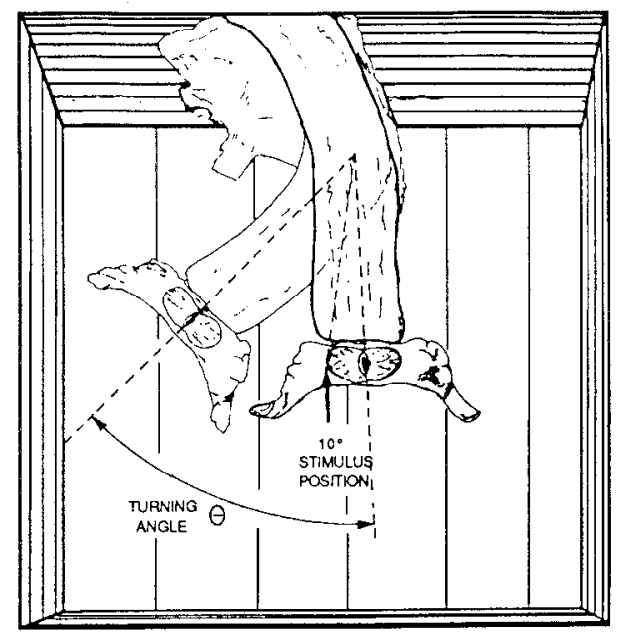

Figure 1. Illustration of Aplysia oriented in feeding posture and performing turning response. The animal (seen from above) has attached itself to the wall of the tank by means of the posterior part of its foot. The anterior part of the foot, including the neck and the head, is free and contacts the surface of the water. In this posture, touching a piece of seaweed on the head of the animal evokes a turning movement in the direction of the stimulus. In the case depicted, the stimulus was positioned at a $10^{\circ}$ eccentric location (vertical arrow) from the midline of the body (dashed line). This evoked a turning response that terminated at a turning angle of about $50^{\circ}$ (only the initial and the final position of the animal is shown). Still video frames were used to measure the turning angle of the animal.

Extracellular recordings. Extracellular recordings were carried out either from pleural and pedal nerves (P11, P4, P5), which innervate neck muscles, or directly from the neck muscles by mean of en passant cuff electrodes. The uninsulated tip of a platinum-iridium wire (Medwire $10 \mathrm{IR} 9 / 49 \mathrm{~T})$ was inserted into a short (2-3- $\mathrm{mm}$ ) piece of Silastic tubing (inside diameter, $0.02 \mathrm{~mm}$ ), which was slit open lengthwise. The cuff was placed either around a nerve close to the ganglion or over a small bundle of muscle fibers separated from the main bundles of the neck muscles. The cuff was insulated and kept in place by cyanoacrylate glue (Krazy Glue). Recordings were also made from the cut ends of nerves that were drawn into small-diameter Silastic tubing. Conventional datarecording techniques were utilized. Simultaneous with the electrophysiological recordings, the animal was monitored with a video system so that the bchavior and the recordcd activity could be correlated.

Kinematic analysis of the head-turning movements. The torque needed to generate head turning was computed on the basis of trajectory data, using a physical model that takes into account the inertia of the turning body, the frictional drag, and the elasticity of the body of the animal. A related approach has been used by Bizzi et al. (1978) to calculate the torque necessary to generate head movements in monkeys. Two simplifications were introduced: First, the anterior (turning) part of the animal's body was assumed to pivot around a point, and second, the elasticity of the body was assumed to be constant. Then, the torque $(T)$ that generates the turning movements of a cylindrical body that resembles that of the animal is given by the time-dependent function.

$$
T(t)=\dddot{I} \ddot{\Theta}+F \dot{\Theta}+E \Theta,
$$

where $\Theta$ is the turning angle (in rad); $\dot{\Theta}$ is the angular velocity; $\ddot{\Theta}$ is the angular acceleration; the moment of inertia of the turning body, $I$, = $1 / 2 M R^{2}(M=$ mass, $R=$ radius of turning object); frictional drag, $F,=$ $3 \pi \mu A V\left(A=\right.$ area, $V^{\prime}=$ velocity, $\mu=$ kinematic viscosity $)$; and $E$ is the elasticity of the bending elements; $t=$ time.

In slow-moving bodies, the frictional drag is determined by the viscosity of the medium rather than by drag forces. Hence, additional frictional cofactors, which, for example, depend on the shape and the surface of the moving body, are extremely small at the low velocities of Aplysia movements and can therefore be neglected. The elasticity $(E)$ of the system consists of passive factors of the tissue and active components, such as the muscle tonus and contractions, and it cannot easily be determined empirically. An estimated value of elasticity, based on measurements in other muscles (see, e.g., Barmack, 1976; Stark et al., 

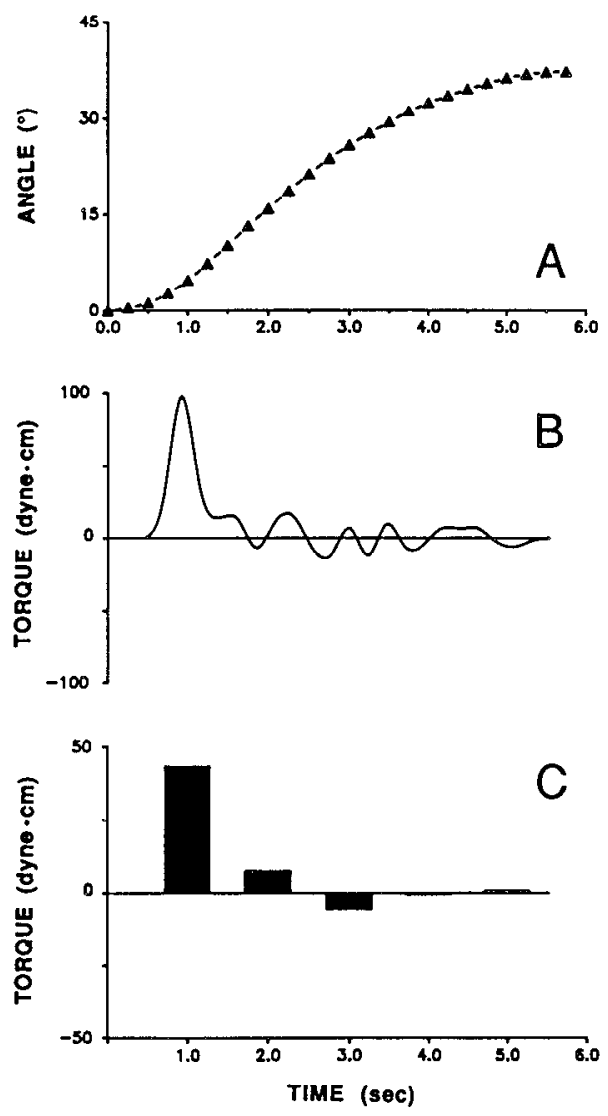

Figure 2. Calculated torque that powers movement of physical model. $A$, Smoothed trajectory of a movement generated by a brief manual push of a water-filled condom. $B$, Calculated torque plotted for each $250-\mathrm{msec}$ time interval. The initial peak reflects the manual push of the system. For the remainder of the movement, no net force is apparent, indicating the passive nature of the swinging system. $C$, Torque averaged per 1 -sec time interval.

1988) was used. Because the elasticity was the only unknown parameter in the equation, this value was systematically varied over 1 order of magnitude and the results compared. $\Lambda$ s we demonstrate below, the value of the elasticity does not qualitatively affect the results, and implications of different values of elasticity on the muscular system will be specifically addressed in the Discussion. The calculation magnifies errors made in the measurements of the turning angle. To minimize the variability, the trajectories of the turning movements were smoothed over 3 data points. These data were then used to calculate the torque per time interval. The results are plotted in bar histograms with upright bars representing a torque in the turning direction and downward-directed bars indicating a torque in the opposite direction, that is, a braking force.

To test the validity of the theoretical approach to an object in an aqueous medium, the above procedure was applied on trajectories derived from a physical system. This model consisted of a water-filled condom (in a shape that resembles the body of an Aplysia) attached to a wall of a tank and floating on the water, so that the experimental conditions were similar to those of the animals. When the condom was stationary in a center position, it was pushed manually for approximately $1 \mathrm{sec}$. Following the push, the movement of the system is exclusively governed by passive factors. Thus, the torque should have a peak at the beginning of the movement for a duration of approximately $1 \mathrm{sec}$, the duration of the push. During the remainder of the movement, the net force should be approximately 0 , because no additional force was applied. Inertia and frictional drag of the model were calculated, and different values of elasticity were assigned to Equation 1 until the torque data yielded a good fit, reflecting the passive nature of the model.

Figure $2 A$ illustrates the smoothed trajectory of a turning curve of such an experiment. The calculated torque per time interval (for the optimal value of elasticity) is plotted in Figure $2 B$; the data, averaged per 1-sec time interval, are plotted in Figure $2 C$. As predicted, the torque has a peak in the initial time interval, representing the force applied to the system by pushing it. In accordance with our expectations, after the initial peak, little or no torque is apparent (the small oscillations represent an expected level of variability, due to errors of measurement). Very similar results were obtained by applying the procedure on trajectories of smaller or larger movements, generated by weaker or stronger pushes. The obtained results appear to describe the expected behavior of the model system and to provide support for the validity of this approach for determining and torque that generates the turning movements of the animals.

Statistical analysis. Two-way comparisons were done using the MannWhitney $U$ test. For multifactor comparisons, analysis of variance (ANOVA) was carricd out using the STATVIEw II (Abacus Concepts) computer program. Data in the form of percent responses or percent of control were adjusted by arcsin or $\log$ transformations, respectively (Winer, 1971).

\section{Results}

In a quiescent animal, a seaweed stimulus initially evokes withdrawal responses. The animal then expands it head, lifts it off the substrate, and assumes a posture characteristic for feeding (see also Preston and Lee, 1973; Kupfermann, 1974a). Subsequent seaweed stimulation evokes turning movements without any indication of withdrawal. Observations revealed that the animal turns in a direction that is determined by the stimulus position on its body surface: Tentacle stimulation results in a lateral turning movement, stimulation of the rhinohpore results in the animal arching backwards, and a stimulus applied to the anterior foot, which lies on the water surface, causes the animal to move its head toward the foot. In other words, the animal moves its head in 3-dimensional space with the locus of the stimulus on the receptive surface determining the vector (direction and magnitude) of the evoked movement.

\section{Overshooting turning responses are evoked by stimuli presented in an open-loop condition}

For a quantitative analysis of the turning responses under openloop conditions, brief seaweed stimuli were presented at various loci on the lips and tentacles along a line through the mouth and orthogonal to the long axis of the body. These stimuli elicit turning movements in the horizontal plane only. Figure $3 A-D$ shows trajectories of turning responses of a typical animal for stimulation at positions of $0^{\circ}, 1^{\circ}, 5^{\circ}$, and $10^{\circ}$ eccentricity. Applying the stimulus directly to the mouth $\left(0^{\circ}\right)$ resulted in small amplitude movements without a clear directional tendency (Fig. $3 \mathrm{~A}$ ). Stimulation at a $10^{\circ}$ position (lip-tentacle boundary) evoked turning movements that were directed toward the stimulated side but terminated at angles of about $50-60^{\circ}$, that is, at a position far beyond the stimulus (Fig. $3 D$ ). To determine if the turning response is a function of the eccentricity as well as the left-right position of the stimulus, the animals were stimulated at $1^{\circ}$ and $5^{\circ}$ eccentricities, and the resulting turning movements were compared to those of the $10^{\circ}$ stimulus. A stimulus applied near the mouth at $1^{\circ}$ eccentricity evoked turning responses that terminated at about $10-15^{\circ}$ (Fig. $3 B$ ), whereas stimulation at $5^{\circ}$ (Fig. $3 C$ ) evoked turning movements to $35-45^{\circ}$. The averaged data of a group of 6 animals for stimulation at $1^{\circ}, 5^{\circ}$, and $10^{\circ}$ eccentric positions (Fig. $4 A$ ) clearly indicate the progressive increase in the final turning angle and velocity of the turning response as a function of increasing eccentricity of the stimulus position. The mean final turning angle is a linear function of the stimulus position within the range studied (Fig. $4 B$ ).

The data of these experiments suggest that the turning re- 

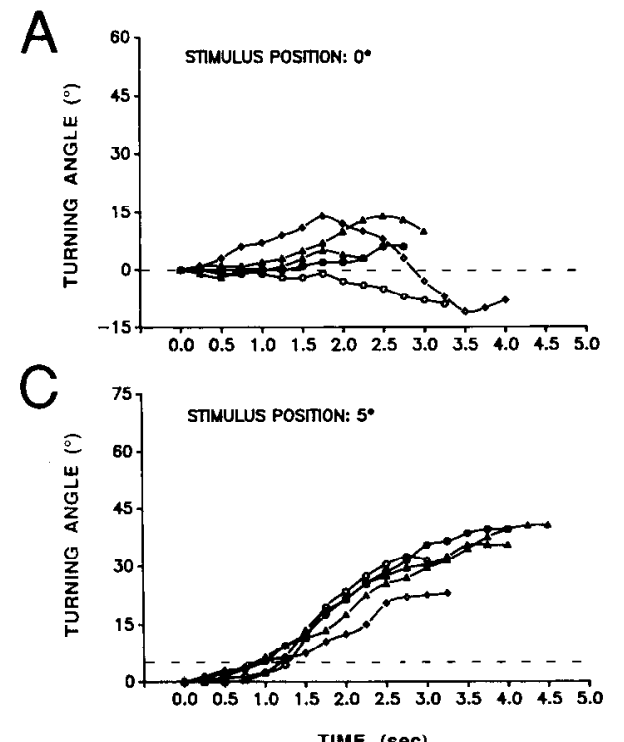

TIME (sec)
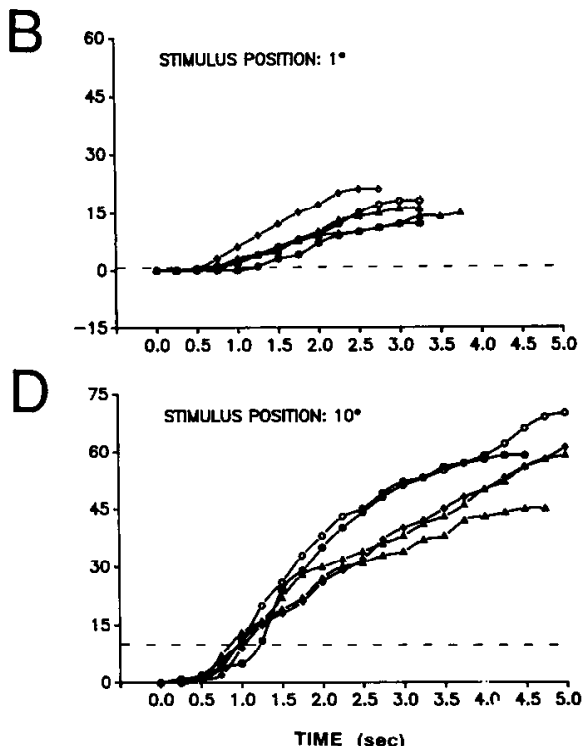

Figure 3. Trajectories of turning responses performed under open-loop conditions, showing trajectories (turning angle in degrees over time) of typical animal performing turning responses following seaweed stimulation at $0^{\circ}(A)$ $1^{\circ}(B), 5^{\circ}(C)$, and $10^{\circ}(D)$ eccentric positions, respectively ( 5 trials each). The stimulus position is indicated by the dashed line. Thus, if the animal moved its mouth to the stimulus, that is, if it centered the food, the trajectory of the turning angle should terminate near the dashed line. sponse of the animal is not only coded by the direction of the stimulus, but in addition, is a graded function of the stimulus position relative to the mouth. The overshooting response of the animal may reflect a central (neural) mechanism that responds differentially to stimuli at different loci. Alternatively, the overshoot might be due to residual chemicals remaining for a period of time in the mucous layer that covers the receptive surface. Although the touch was brief, remaining residues of the seaweed could provide prolonged stimulation. Arguing against this possibility is our finding that the response is graded according to the stimulus position. To more directly determine the role of residual chemical stimuli, we evoked turning responses by using a purely tactile stimulus instead of the seaweed stimulus (which has both tactile and chemical components).

\section{Tactile stimulation is effective in eliciting a turning response,}

\section{but the response decrements}

Tactile stimulation evokes head-turning responses in foodaroused animals as reliably as seaweed stimulation (see also Preston and Lee, 1973), and like the responses to seaweed, the tactually evoked responses also overshoot the stimulus position.
However, whereas the turning responses to repeated seaweed stimulation remained relatively constant in magnitude, the response magnitude upon repeated tactile stimulation decremented rapidly. After 5 or 6 tactile stimuli, the animals generally ceased to respond. To evalute the differences in the turning responses triggered by the 2 stimulus modalities, 5 animals were stimulated repeatedly (every $10 \mathrm{sec}$ ), either with a glass probe (a pure tactile stimulus) or with seaweed (a chemical plus tactile stimulus), both applied at the same $10^{\circ}$ spot on the lips. We found a significant effect of the stimulus modality on the response magnitude $[F(1,8)=17.2, p<0.005]$. Repeated tactile stimulation lead to a rapid decline in response magnitude, whereas repeated seaweed stimulation (Fig. 5) evoked relatively constant responses [2-way interaction: $F(7,56)=4.2, p<0.005$ ]. Decrementing responses were also observed for tactile stimulation of other parts of the animal, such as the rhinophores.

\section{Purely chemical stimuli are much less effective than purcly tactile stimuli in eliciting a turning response}

To determine the role of the chemical component of the seaweed stimulus in eliciting the response, food-aroused animals were

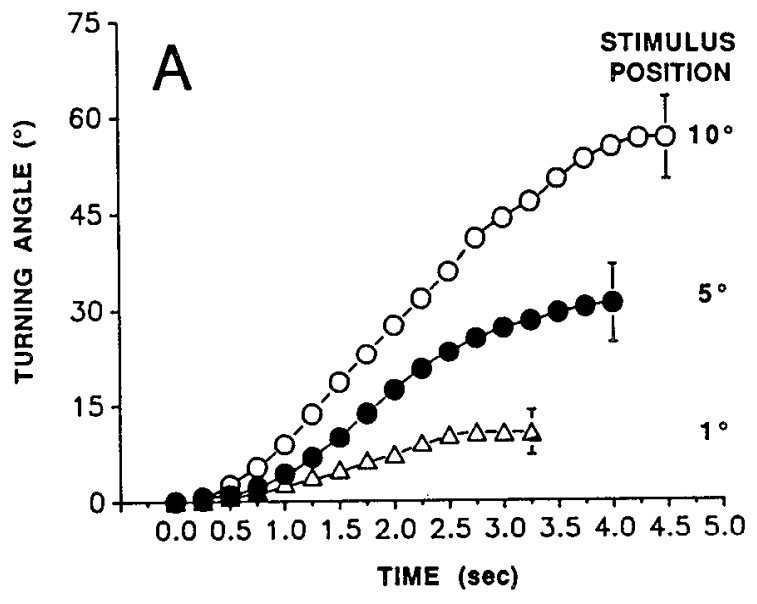

TIME (sec)

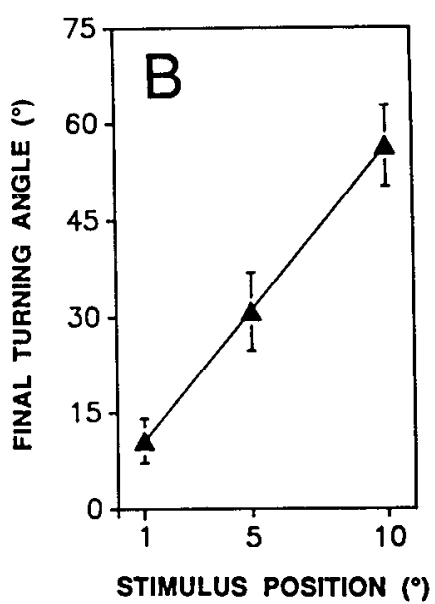

Figure 4. Magnitude of turning response under open-loop conditions is graded according to stimulus eccentricity. $A$, Averaged turning responses of a group of 6 animals to stimulation at $1^{\circ}$, $5^{\circ}$, and $10^{\circ}$, respectively ( 5 trials each). Vertical bars at the terminal points give SEM. $B$, Average final turning angle ( \pm SEM) of turning responses for the different stimulus positions. Note the linear relationship of the final turning angle to the eccentricity of the stimulus. 


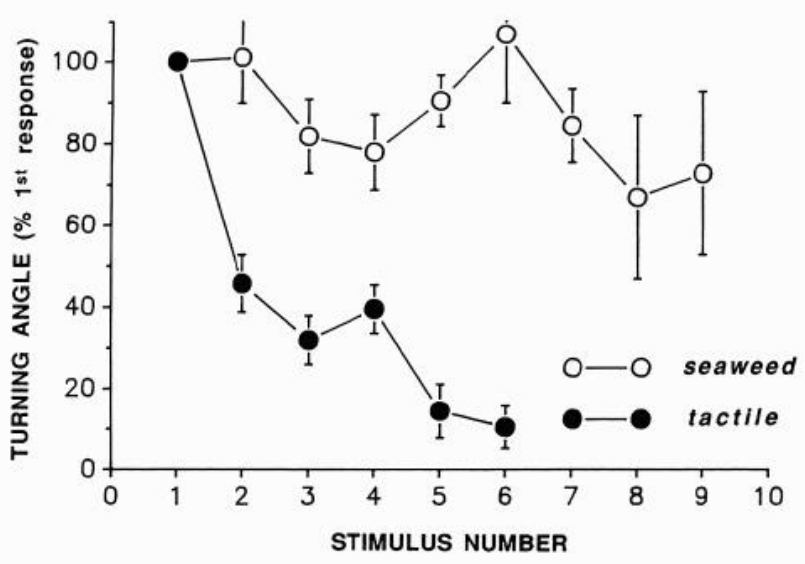

Figure 5. Repeated tactile stimulation leads to decrementing turning responses in contrast to repeated seaweed stimulation. The animals $(n$ $=5$ ) were stimulated either with a purely tactile stimulus (solid circles) or with seaweed (open circles) at a $10^{\circ}$ locus, and the stimulus was repeated every $10 \mathrm{sec}(10$ successive stimuli, 3 series each). Final turning angles of the responses are shown as percent of the final angle of the first response (means $\pm \mathrm{SEM}$ ). Note the marked decline in the magnitude of the turning response evoked by repeated tactile stimulation and the relatively steady response magnitude upon repeated seaweed stimulation.

stimulated either with a purely tactile stimulus (glass probe) or with a purely chemical stimulus, which consisted of a solution of seaweed extract that was slowly released out of a pipette near the tip of the tentacle. Solutions were colored with a dye (fast green) so that it was possible to observe the spread of the solution and to judge if the stimulus had actually reached the tentacle of the animal. As a control for unspecific effects of the chemical stimulus, seawater (dyed with fast green) was released in a similar fashion near the tentacle.

Tactile stimuli were highly effective in eliciting turning responses (Fig. 6), whereas the control (seawater) hardly elicited any responses, and pure chemical stimulation only occasionally

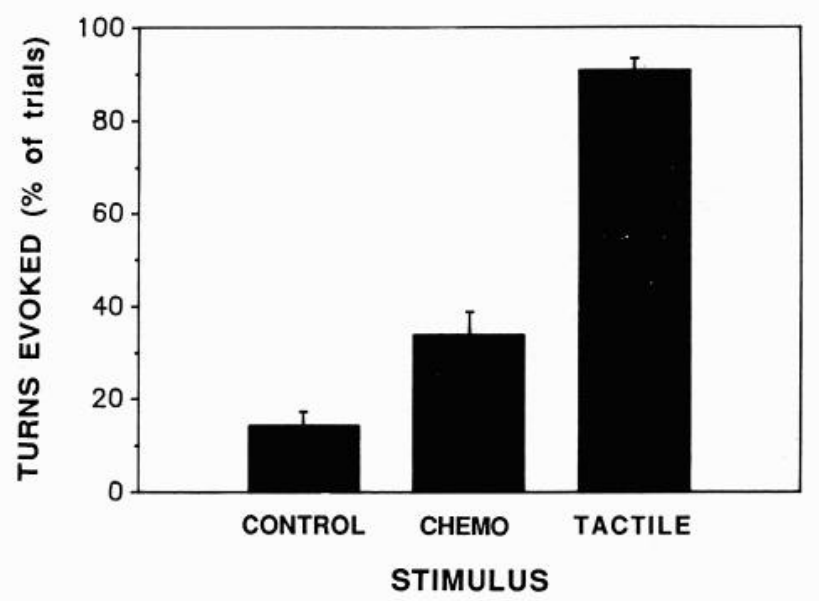

Figure 6. Purely chemical stimulus is less likely to evoke turning response than purely tactile stimulus. On separate trials, food-aroused animals $(n=9)$ were each stimulated either with a purely chemical stimulus (seaweed extract slowly ejected out of a pipette near the tip of the tentacle) or with a tactile stimulus (glass rod) on the tentacle (10 trials each). To determine baseline response rates and to test for unspecific effects of the chemical stimulation, artificial seawater was perfused near the tentacle for 1 set of trials.

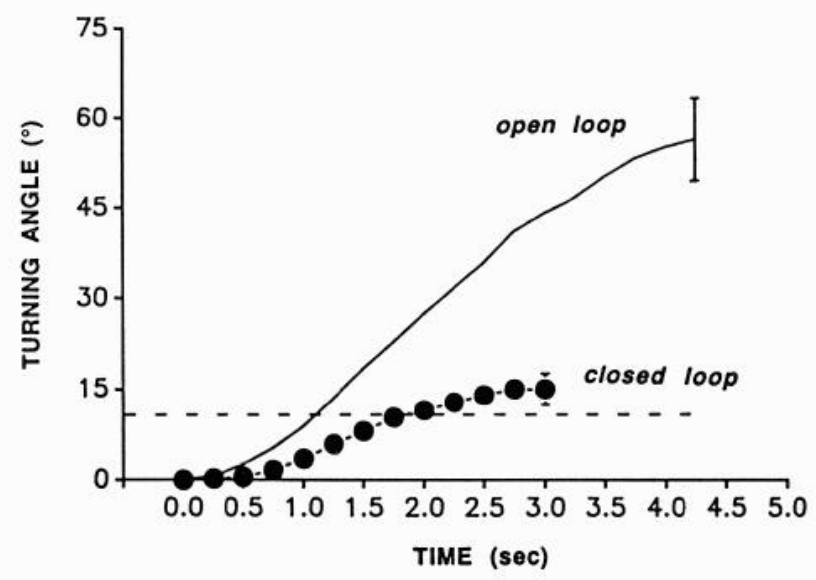

Figure 7. Turning responses evoked in closed-loop conditions are more accurate than those under open-loop conditions. Circles represent averaged data of 5 animals stimulated under closed-loop conditions ( 5 trials each); the SEM is very small for the individual data points and is only plotted for the terminal point. The solid line represents averaged data for responses evoked under open-loop conditions (taken from Fig. $4 A$ ). Under both conditions, the stimulus was initially applied at $10^{\circ}$ eccentricity (dashed line). Note that the turning movements of the animals under the 2 different experimental conditions appear to differ relatively early during the movement.

elicited turns [overall effect: $F(2,10)=38.2, p<0.001$ ]. Individual Scheffé $F$ tests revealed that tactile stimuli were significantly more effective than chemical and control stimulation [control vs tactile: $F(1,8)=35.1, p<0.001$; chemical vs tactile: $F(1,8)=20.3, p<0.001]$, whereas the slightly higher efficacy of chemical stimulation compared to control was not significant $[F(1,8)=2.0, p>0.20]$. Thus, the tactile component of a seaweed stimulus is of paramount importance as a trigger for the turning response, even though it loses its effectiveness when repeatedly presented. Chemical stimuli alone elicit few or no responses.

\section{Closed-loop conditions enable centering of the food}

Our initial experiments were performed under open-loop stimulus conditions, in which the stimulus was applied only briefly at a defined spot. Under these conditions, the animals performed poorly in orienting to the point in space where the stimulus was presented. A more accurate orientation could require closedloop conditions, in which the animals receive continuous stimulus feedback while turning. Indeed, we found that closedloop conditions resulted in far more accurate turning movements. In Figure 7, the trajectory of the turning responses to a $10^{\circ}$ stimulus under closed-loop conditions is plotted in comparison to the previously plotted trajectory to $10^{\circ}$ stimulation under open-loop conditions. Instead of the relatively high velocity and overshoot that characterized the responses upon brief stimulation, the turning responses upon closed-loop stimulation started out more slowly and terminated when the mouth of the animal was close to the food. Animals either turned accurately or slightly overshot and then turned back.

In analyzing the mechanisms underlying the more accurate turning in the closed-loop condition, at least 3 possibilities must be considered: (1) Under the closed-loop conditions (but not under open-loop), there is the possibility of adjustment after overshoot. The animal could turn as if stimulated in an openloop fashion, thus overshoot so that the opposite lip contacts the food, and it could then reverse its turn. This pattern could 


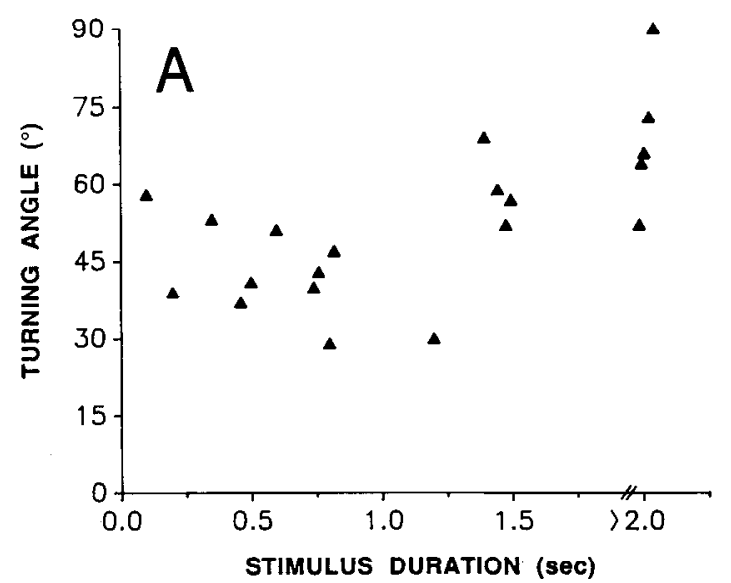

be repeated with successively smaller overshoots until the mouth of the animal is centered at the stimulus. (2) The magnitude of the response could be negatively correlated with the duration of the stimulus, and therefore, because under closed-loop conditions the stimulus is present for a long duration, it would evoke small turns. (3) The movement of the stimulus along the receptive field might influence the turning movements. The experiments performed under open-loop conditions showed that medial stimulation elicited smaller responses than lateral stimulation. Therefore, it is conceivable that medial stimulation nullifies the stronger response to a preceding lateral stimulus and evokes a turning response to a smaller turning angle. Consequently, under closed-loop conditions, as the stimulus moves from lateral to more medial receptive fields, the smaller response elicited by more medial stimulation might substitute for the larger response elicited by the preceding lateral stimulation. This will be referred to as the stimulus substitution hypothesis. The 3 possible mechanisms were explored in experiments described in the following sections.

\section{Accurate turning is not due to overshoot and adjustment}

If the animal turns accurately under closed-loop conditions by starting a typical open-loop turn, and as soon as the opposite lip touches the food, the animal initiates a reverse movement, then the early parts of the turning movement should be very similar under both conditions. Figure 6, however, indicates that the mean trajectory of the turning response under closed-loop conditions accelerates more slowly than that obtained under open-loop conditions. We measured the mean time for the animals to turn a fixed amount $\left(8^{\circ}\right)$, for turning movements under open- and closed-loop conditions. We chose an $8^{\circ}$ turn, which is still $2^{\circ}$ from the center position (initial stimulus position was $10^{\circ}$ ), so as to guarantee that the stimulus did not touch the opposite side of the mouth. Under closed-loop conditions, it took the animals an average of $1.6 \pm 0.2 \mathrm{sec}( \pm$ SEM) to perform an $8^{\circ}$ turn. Under open-loop conditions, the animals reached the same angle after a significantly shorter time of $1.2 \pm 0.07$ sec (Mann-Whitney $U$ test; $p<0.05$ ); or conversely, under open-loop conditions, the animals turned $17 \pm 2.0^{\circ}$ in $1.6 \mathrm{sec}$, the amount of time in which they turned only $8^{\circ}$ when stimulated under closed-loop conditions. This suggests that the centering of the mouth to the food, observed under closed-loop conditions, is a result of a fundamentally different turning response, which is considerably slower in all phases of the movement than the response under open-loop conditions.
Accurate orientation in closed-loop conditions is not due to the prolonged presence of the stimulus

To test whether prolonged stimulation can account for the smaller response under closed-loop conditions, we performed experiments in which the stimulus position was kept constant and the duration of the stimulus was varied. Food stimuli were applied at a $10^{\circ}$ position and kept in place for durations between $100 \mathrm{msec}$ and $5 \mathrm{sec}$. The duration of the stimulus was measured by a stop watch, displayed in the video image. The final turning angle of responses evoked by stimuli of different durations is plotted for a typical animal in Figure $8 A$. There was relatively little influence of the duration of the stimulus on the final turning angle for stimulus duration between 100 and $1500 \mathrm{msec}$; stimuli exceeding $2 \mathrm{sec}$ in duration evoked stronger turning responses of the animal. Figure $8 B$ illustrates the average final turning angle as a function of stimulus duration for 3 experimental animals. Stimulus durations of up to $1.5 \mathrm{sec}$ had relatively little effect on the magnitude of the turning response, and the average turning angle was similar to those measured in previous experiments for $10^{\circ}$ stimulation (see Fig. 4B). Stimuli applied for longer than $2 \mathrm{sec}$ evoked a larger turning response. These data indicate that a prolonged presence of the stimulus cannot account for the smaller responses under closed-loop conditions, because longer contact with the stimulus causes stronger rather than weaker responses.

\section{Responses to medial stimulation can substitute for those to lateral stimuli}

The difference in the responses under closed- and open-loop conditions might be due to movement of the stimulus along the receptive surface when stimulated under closed-loop conditions. This could create a situation where a strong response to a lateral stimulation becomes substituted for by a weaker response to more medial stimulation. To gain information about the existence of such a mechanism, we performed experiments in which a lateral $\left(10^{\circ}\right)$ stimulus was followed by a medial stimulus $\left(5^{\circ}\right)$. Two trajectories of turning responses of a typical animal tested according to the above protocol are shown in Figure 9.4 (solid symbols), together with 2 control responses evoked by a single $10^{\circ}$ stimulus (open symbols). In the 2 cases in which a second stimulus was applied (time indicated by arrowheads below the curves), the animal did not complete its turning movement to $50-60^{\circ}$ (expected turning angle for $10^{\circ}$ stimulation), but terminated the turn at a reduced angle of only $20-25^{\circ}$. If a total 

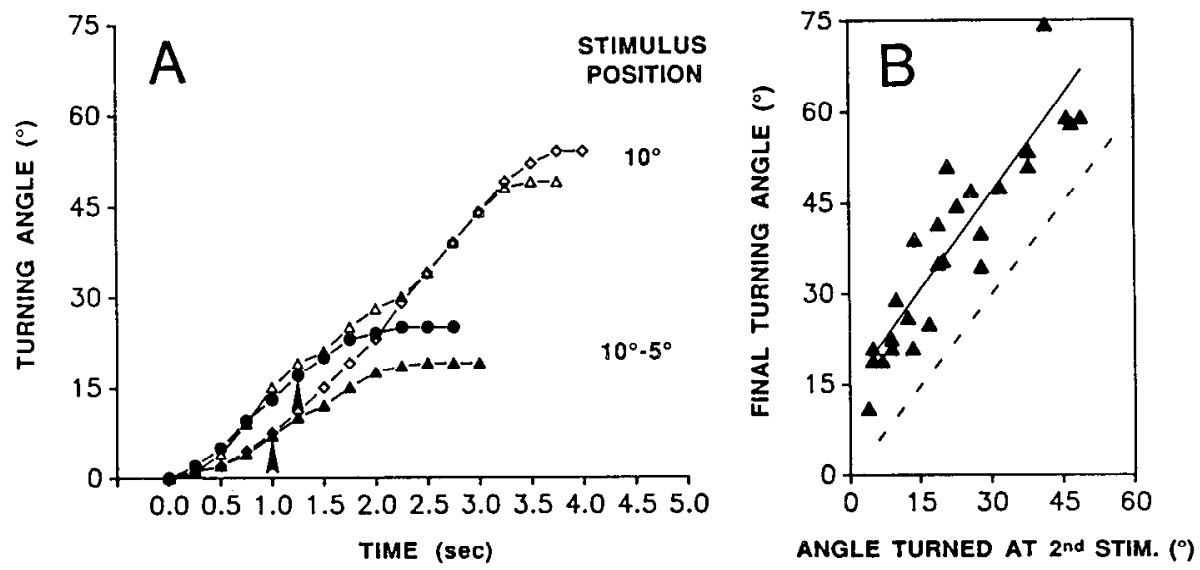

Figure 9. Turning responses evoked by lateral stimulation can be affected by subsequent medial stimulation. $A$, Trajectories of 2 turning responses of a typical animal stimulated at a $10^{\circ}$ locus (open symbols), and when also stimulated at $10^{\circ}$, but followed (during the movement) by a more medial stimulus on the same side of the mouth at approximately $5^{\circ}$ (solid symbols). The presentation of the second stimulus is indicated by arrowheads. $B$. Final turning angles of responses triggered by $10^{\circ}$ stimulus, followed by a $5^{\circ}$ stimulus at a time when the animals $(n=6,5$ trials each) had already turned to a given angle (plotted in the $x$-axis). The dashed line indicates the angle at which the animals should have stopped turning if the second stimulus were to immediately stop the movement. Note that the regression line (solid line) of the final turning angles of the animals is almost parallel to the dashed line.

substitution of the response occurs, one would predict that the second (medial) stimulus would evoke a turning response to a certain angle that is independent of the angle turned at the time of stimulation. We therefore stimulated at $10^{\circ}$ and followed it by a $5^{\circ}$ stimulus, which was presented after the animals had turned varying amounts in response to the first stimulus. In Figure $9 B$, final turning angles $(n=6,5$ trials each) are plotted as a function of the angle turned at the time when the second stimulus was applied. The dashed line indicates the angles at which the animals would have stopped if the application of the second stimulus were to immediately abort the ongoing movement. The data show, however, that the animals continued their turning movements to a certain extent before coming to a halt. As indicated by the regression line, the animals turned an additional $15^{\circ}$, regardless of whether they were stimulated at the beginning of their turning movement or when they had almost completed the initial movement. This $15^{\circ}$ turning movement is consistent with the hypothesis that the medial stimulus substituted a weak response for the stronger response to the first, more lateral stimulus.

However, another interpretation of these results is possible. The medial stimulation may have caused a termination or braking of the ongoing response, and the $15^{\circ}$ turn was due to the inertia of the turning body. One reason why medial stimuli might engage a braking mechanism is that medial (but not lateral) stimuli are more likely to elicit biting responses, and the biting program might be associated with the generation of a braking force. Because we have not been able to quantitatively vary the angular position of the second stimulus in order to test whether different loci of secondary stimulation leads to different amounts of additional turning movements, it has not been possible to directly test the stimulus substitution hypothesis. We have, however, been able to explore the possible role of biting on the turning responses.

\section{Biting responses do not influence ongoing turning movements}

Biting responses have been observed under both open- and closed-loop conditions and have occurred most often when the stimulus was applied near the mouth. The relation between stimulus position and the percentage of stimuli cvoking bitcs is plotted in Figure $10 \mathrm{~A}$. Stimulus locations at $0^{\circ}$ and $1^{\circ}$, that is, in the direct vicinity of the mouth, evoked biting responses very effectively; stimulating at $5^{\circ}$ evoked bites in about $50 \%$ of the trials, and stimulation at $10^{\circ}$, or further eccentric on the tentacles or rhinophores, practically never evoked a bite. Thus, the capacity of a stimulus to elicit a bite declines with increasing stimulus eccentricity.

If there is an interruption of the turning motor program due to the biting program, one would expect that turning responses evoked by stimulation at a similar locus should differ in their final turning angle as a function of whether or not a bite occurred. This was tested by comparing the final turning angles of the responses upon $5^{\circ}$ stimulation (which evoked bites in about 50\% of the trials) in the absence or presence of accompanying bites. The mean final turning angle of turning movements (5 turns in 4 animals) was $30 \pm 9^{\circ}( \pm$ SEM $)$ when they were accompanied by bites. When no bite occurred, the mean final turning angle was $27 \pm 10^{\circ}$, which is not significantly different (Mann-Whitney $U$ test, $p>0.5$ ) from the angle when the animals bit, suggesting that there is no direct interaction between the bites and the turning movements of the animals.

Analysis of the video recordings of turning responses indicated that the biting responses occurred mostly during the late phase of the turning movements. Therefore, the biting response to $5^{\circ}$ stimulation might occur too late to influence the turning movement, but more central stimuli might trigger bites with shorter latencies. However, we found that the average bite latencies for stimulation directly on the mouth $\left(0^{\circ}\right)$ and at either $1^{\circ}$ or $5^{\circ}$ were similar (about $3 \mathrm{sec}$; Fig. 10B). Thus, a more medial stimulus does not elicit bites sooner than a more lateral stimulus, which makes it unlikely that the generation of a bite is responsible for braking the turning movements. Although biting responses do not appear to be associated with an inhibition of ongoing movements, in the following section we provide evidence that the presence of a bite suppresses the capacity of subsequent stimuli to elicit a head-turning response. 

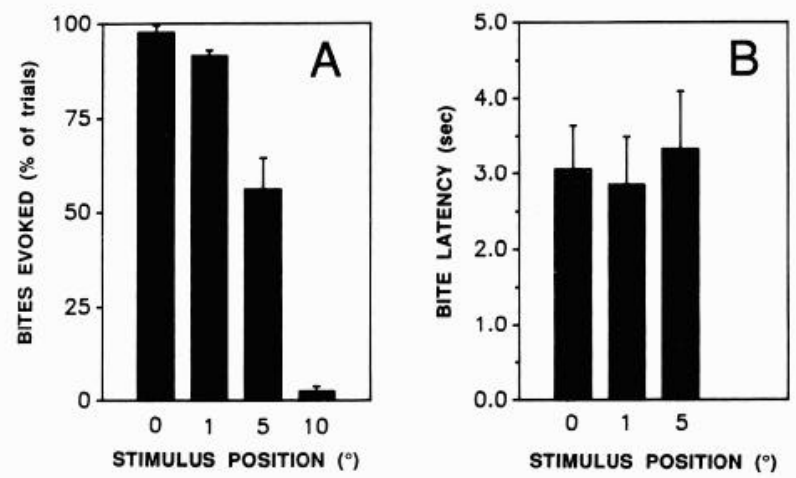

Figure 10. Position of stimulus relative to mouth determines its capacity to trigger bites. $A$, Mean probability ( \pm SEM) of a biting response that occurs within $10 \mathrm{sec}$ following stimulation with seaweed at different eccentricities on the lips and tentacles ( $n=6,20$ trials each). $B$, Average bite latency $( \pm$ SEM) for bites triggered by stimulation at the different eccentricities ( $n=4,10$ trials each). The bite latency is defined as the time interval between application of the stimulus and the maximum protraction of the radula.

\section{Biting responses gate out turning responses}

For quantitative examination of the conditions under which the animals orient or do not orient to stimulation with seaweed, we first tested animals under 3 different behavioral activities: (1) when they were quiescent, (2) while performing spontaneous turning movements, and (3) while exhibiting spontaneous biting responses. Figure 10 shows the mean stimulus efficacy for 6 animals tested under the 3 conditions ( 25 trials each). A test stimulus was highly effective in eliciting a turning response when the animals either were quiescent $(90 \%)$ or were turning spontaneously $(100 \%)$. In contrast, the efficacy of the stimulus was reduced to $5 \%$ during periods when the animals were spontaneously biting. Similar results were obtained for animals engaged in triggered rather than spontaneous behaviors. When the animals performed evoked turning movements, stimulation on the opposite side was able to interrupt and reverse the movement. No second turning response was observed, however, if the animal performed a bite together with the initially evoked turning movement.

For a detailed analysis of the gating out of the response during biting, the animals were tested during different phases of bites. A schematic diagram of the temporal sequence of the biting response, beginning with lip closing, followed by protraction and retraction of the radula, is shown above the graphs in Figure 11. The histograms below give the percentage of effective test stimuli, separated according to the time in the biting program when the test stimulus was applied. It can be seen in Figure $12 \mathrm{~A}$ that the test stimulus was totally ineffective (no turning responses observed) during the interval that corresponds to the lip-closing phase (0-2 sec) and was only slightly more effective (turning elicited in $8 \%$ of the trials) during the protraction phase of the radula (2-3 sec after initial stimulus presentation). In the later phase of the biting program, when the radula is in the process of being retracted (3-4 sec after initial stimulus), the animals responded in $95 \%$ of the cases to the test stimulus. If the animals performed multiple bites (i.e., a second bite occurred immediately following the first bite), then they did not respond during the first retraction phase, either (not illustrated). This suggests that the inhibition may be related to the generation of

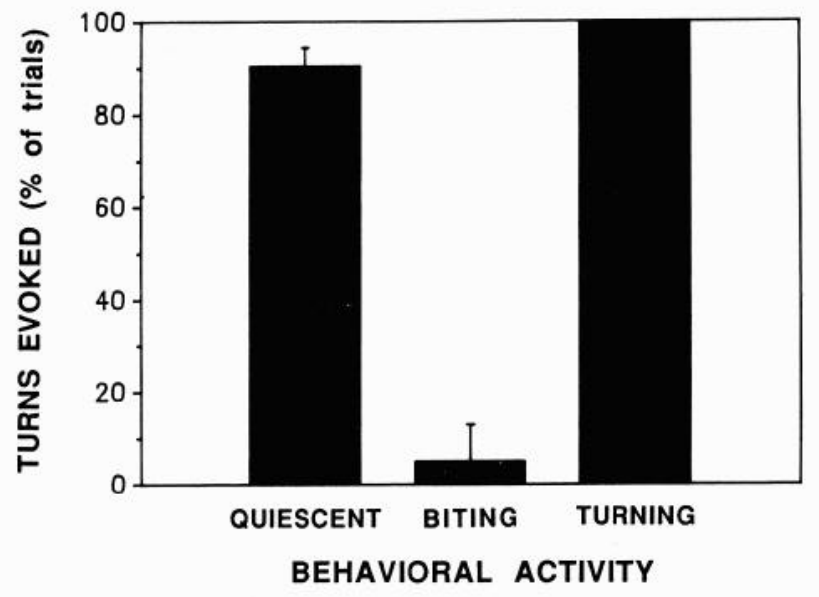

Figure 11. Spontaneous bites gate out turning responses. The capacity of a food stimulus to evoke a turning response was tested during different spontaneous behaviors in food-aroused animals $(n=6)$. A test stimulus was delivered on the tentacle when the animal was quiescent, during spontaneous bites, and while performing spontaneous turning movements ( 20 trials each). Note that the probability of evoking a turning response was greatly reduced during the execution of spontaneous bites. Vertical lines represent SEM.

a bite command rather than to the activity of buccal muscles, buccal motor neurons, or elements of the pattern generator.

The hypothesis that the bite command is the source of the inhibition was tested by studying animals with bilateral lesions of the cerebral-buccal (C-B) connectives. Previous studies have shown that lesions of the C-B connectives completely abolish biting responses, without affecting appetitive behaviors (Kupfermann, 1974b). Although no bites can be observed, bite command elements can be activated in the absence of the buccal ganglion (Rosen et al., 1988), and bite-related behaviors, such as the closing of the lips, occur. Lesioned animals were stimulated directly on the mouth (perioral zone), a region that in normal animals very effectively triggers biting responses (see Fig. 10A). As shown in Figure 12B, the efficacy of the test stimulus in the lesioned animals was also reduced during the 2 initial phases when the bite would have occurred (0-2 and 2-3 sec), though the inhibition appears to be less prominent than in the intact animal. The fact that the responses were gated out in the lesioned animals indicates that the inhibition is not generated by feedback from the pattern generator or motor neurons in the buccal ganglion. The greater number of responses in the lesioned animals compared to normal animals might be due to the occasional failure of the perioral stimulus to trigger a cerebral bite command despite the high efficacy of such a stimulus in normal animals.

\section{Turning movements are generated by a brief power phase}

As a first step toward an analysis of the neural mechanisms underlying directed head turning, we computed the force (torque) needed to generate the different movements that the animals perform under open-loop conditions using a physical model (see Materials and Methods). The results of the calculations (based on turning responses of 1 animal evoked by stimulation at $1^{\circ}$, $5^{\circ}$, and $10^{\circ}$; see Fig. $3 B-D$ ) are shown in Figure $13, A-C$. As can be seen in the figure, the overall timing of the applied torque is similar, regardless of the movement of the animal. Only the first $2 \mathrm{sec}$ of the movements (corresponding to about half the 

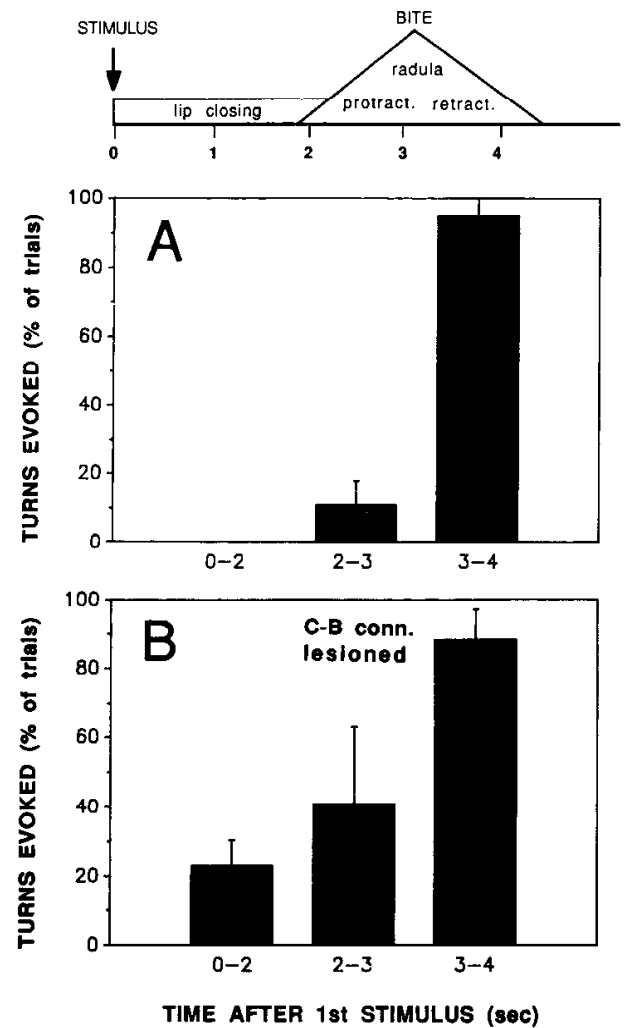

Figure 12. Gating of turning response does not require feedback from buccal ganglion. The top line is a schematic representation of the temporal sequence of the biting response: lip closing and radula protraction and retraction. Biting responses were triggered by a seaweed stimulus applied to the perioral zone. The corresponding percent of trials in which a turn was evoked by a second test stimulus on the tentacle is shown in the graphs below. A, Efficacy of a food stimulus to evoke a turning response ( $n=4,20$ trials each) during different phases of the biting response (means $\pm \mathrm{SEM}$ ). $B$, Efficacy of a food stimulus in animals with bilateral lesions of the C-B connective ( $n=3,20$ trials each). The stimulus was presented at various times following a food stimulus applied to the perioral zone. Note that the lesioned animals, though they cannot perform biting movements, are also characterized by a gating out of the turning response during the projected time of lip closure and protraction.

duration of the total turn) are powered whether the animal turned to $10^{\circ}, 40^{\circ}$, or $60^{\circ}$. To turn the animals to the different final angles, the amplitude of the applied force differed: According to the simulation, about twice the amount of torque was required to turn the animal to an angle of $60^{\circ}$ (Fig. $13 C$ ) than to turn to $40^{\circ}$ (Fig. 13B). In some instances, the power phase was followed by a brief period during which little or no force should be required to sustain the movements (see Fig. 13A,C), suggesting that the body was swinging passively. In most cases, the simulation revealed negative values of torque just before termination of the movements, indicating a torque in the direction opposite the movement, that is, a braking force. The occurrence of a braking force is the major difference between the active turning movements of the animals as opposed to the passive nature of the model system discussed in Materials and Methods. A braking force may explain the more rapid termination of the movement of the animal, compared to that of the model system.

The final part of the turning movement is governed predominantly by the elasticity of the system. Calculations of the torque
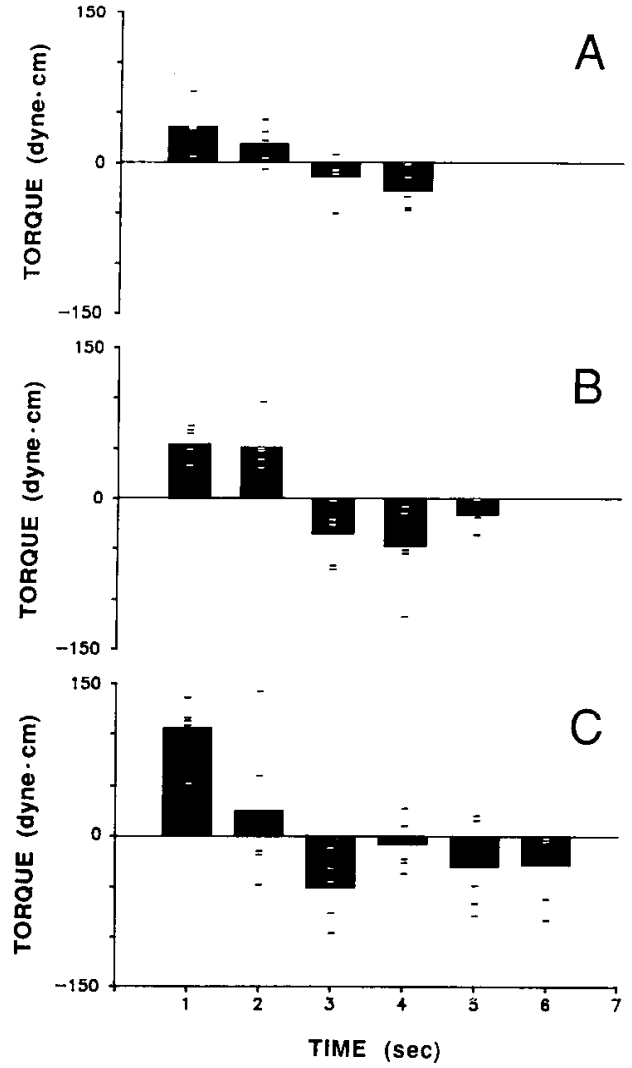

Figure 13. Calculated torque to power turning movements of animal. The torque necessary to generate the turning movements was calculated by applying Equation 1 (see Materials and Methods) to the data of individual turning movements (shown in Fig. $3 B-D$ ), which terminated at $10-15^{\circ}(A), 35-45^{\circ}(B)$, and $50-60^{\circ}(C)$, respectively. Bars give the averaged torque $1-\mathrm{sec}$ time interval; individual data points are indicated by the dashes. The calculation revealed that, in each case, the torque to gencratc the turning movements is generated only during the first 2 sec of the movements, and that, for different movements, the amplitude of the torque varied. At the end of the movement, a force in the negative direction, that is, a braking force, is indicated

using different (constant) values of elasticity, however, revealed that it was not possible to explain the measured trajectories of the turning movements without postulating an active braking force. Furthermore, a constant value of elasticity would not only brake the movements, but would automatically force the animal on a backward-directed course, as is the case for the passive model system. Therefore, it is more likely that the animals adjust the elasticity of their body (their overall body tonus) during and, in particular, at the end of the turn to brake the movements.

The torque data obtained are thought to reffect the neural output to the neck muscles, the muscles involved in the generation of the turning movements. Based on our results, we made 2 predictions about the neural activity necessary to generate the turning movements: First, the neural activity should peak during the first $2 \mathrm{sec}$ of the turning movements, and second, an increase in activity in contralateral nerves should occur at the end of the movements, so as to provide the braking force to terminate the movements. To test these hypotheses, we recorded extracellularly from different pleural and pedal nerves and directly from the neck muscles. 


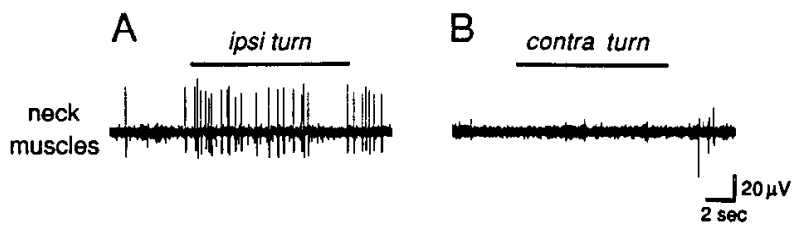

Figure 14. ECs occur in neck muscles during turning movements. Note that, when the animal was turning in the ipsilateral direction $(A)$, more ECs occurred during the initial part of the movement than during the latter part, and no ECs were recorded when the animal turned towards the contralateral side $(B)$.

\section{Extracellular recordings support the prediction of a brief power phase}

Extracellular nerve recordings from different pleural and pedal nerves $(n=6)$ that innervate the neck muscles and directly from the lateral neck muscles $(n=3)$ showed increased unit activity in several nerves and muscles during turning movements (see also Bablanian et al., 1987; Cook and Carew, 1989). Other behaviors of the animal, such as head lifting, were not associated with a significant increase in activity in these nerves and muscles, but contractions of the head and neck caused a substantial increase. This indicates that the muscles recorded from are mostly involved in lateral but not vertical movements of the neck. Figure 14 shows recordings of extracellular currents (ECs) of the lateral neck muscles for an animal turning in the direction ipsilateral or contralateral to the side of the recording electrode. As shown, during ipsilateral turns (Fig. 14A), ECs occur almost throughout the turn, but are more frequent at the beginning of the movement. A turning movement in the direction contralateral to the electrode (Fig. 14B) was not associated with ECs. To quantify these results, we counted the number of ECs that occurred within the first and the second half of 15 ipsilateral and 15 contralateral turning movements of 1 animal (turning movements of different magnitudes were combined). In the ipsilateral direction, about twice as many ECs occurred within the first half of the movement than in the remaining time (110 vs 58 ). None of the turns in the contralateral direction were associated with FC. events. These results provided support for the notion of an early, transient power phase that drives the ipsilateral turning movements. Similar data were obtained in 2 other animals; in another animal, the muscle electrode did not pick up signals when the animal turned left or right, but only when it moved its head backwards, suggesting that the electrode may have been placed relatively medially.

Recordings from individual muscles were not particularly well suited for quantitative data analysis, because relatively few events occurred during the timing movements. Therefore, in order to monitor the activity associated with larger parts of the neck musculature, we recorded from pleural and pedal nerves (Pl1, $n=3 ; \mathbf{P} 4, n=2$; P5, $n=3$ ). In Figure 15 , nerve recordings from different nerves (in different animals) are shown for turning movements of similar magnitude and velocity. During turning movements in the ipsilateral direction (Fig. 15A), we consistently found an increase in unit activity in nerves Pl1 and P5 just before and during the initial phase of the turning movement, and the activity returned to baseline for the remainder of the movement. In nerve P4, several bursts of activity occurred during the course of the turn, with an initial burst that coincided with the beginning of the turn (dotted line). The turning movement in the contralateral direction (Fig. 15B) was associated with a slight decrease in activity in nerve $\mathrm{P} 5$, which then showed an increase in activity during and after termination of the movement. A more prominent increase occurred during the second half of the turning movement in nerve P4, whereas activity of nerve Pl1 was unchanged during the turning movement. The general trend of these patterns holds for all turning movements, except for those where the animal turned in several steps (stop and go) to its final position. In those movements, several bursts of activity occurred ipsilateral, appearing in association with the repeated accelerations of the animal. In contralateral nerves, unit activity could not be correlated to particular phases of those turning movements.

The results of the extracellular recordings suggest that headturning movements of Aplysia are generated by a complex interaction of several muscle groups in different areas of the neck, which are innervated by different nerves. To get an idea of the overall activity, we summed the activity recorded in the 3 nerves (shown in Fig. 15) per 1-sec time interval for the ipsilateral and

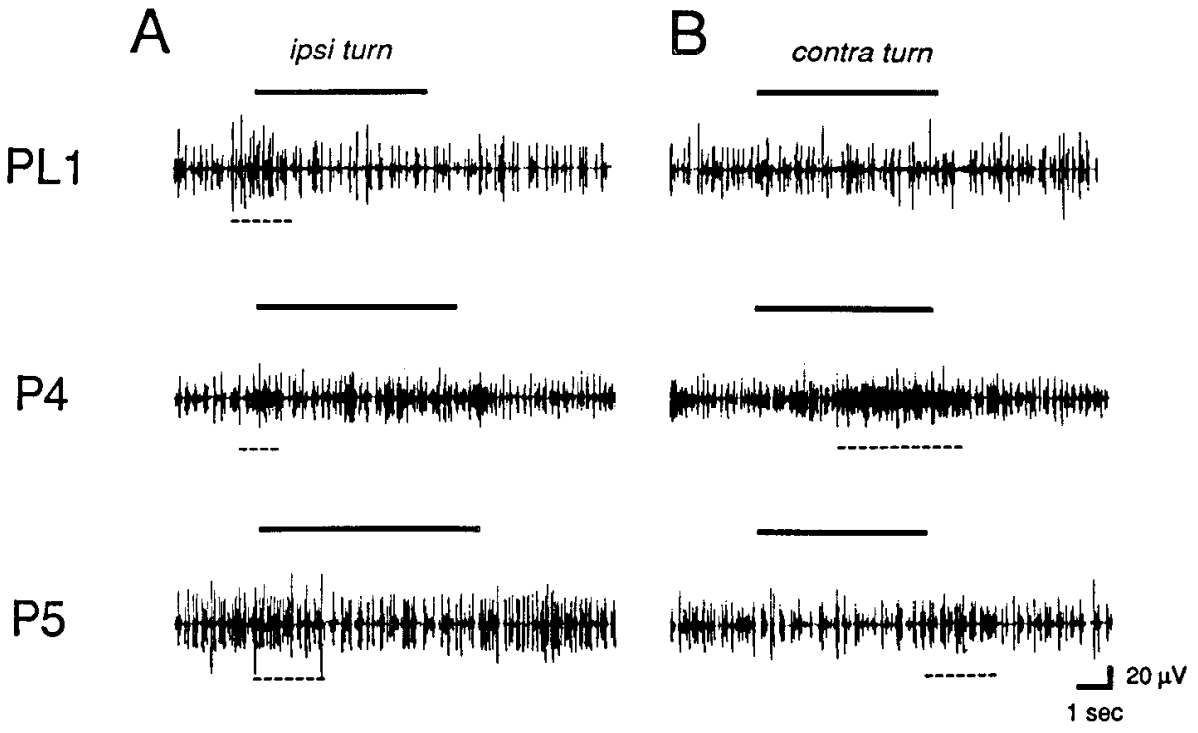

Figure 15. Nerve activity during turning movements. $A$, Recordings from pleural $(P l I)$ and pedal nerves $(P 4, P 5)$, which innervate the neck region of the animal, during turning responses in the ipsilateral direction (recordings from different animals). Duration of the turning movement is indicated by the bars; dashed lines below indicate nerve activity referred to in the text. $B$, Same as in $A$ for turning responses in the contralateral direction. 


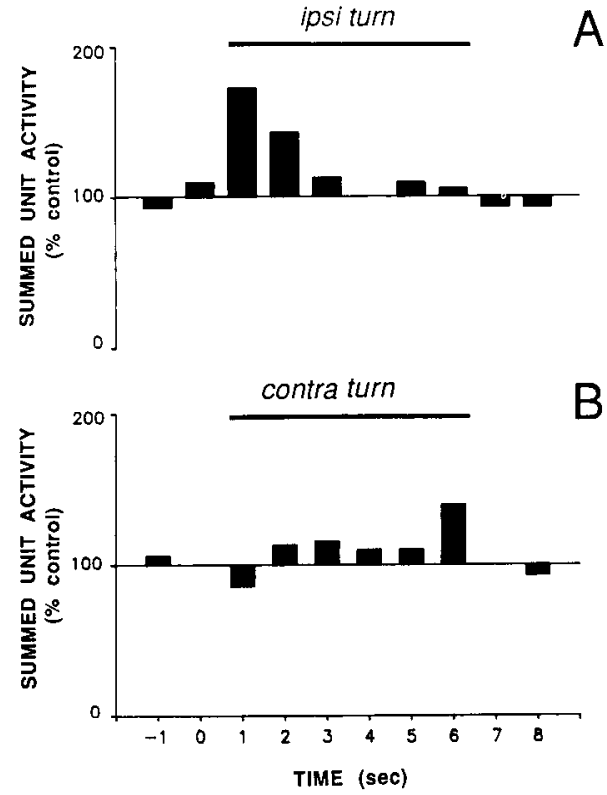

Figure 16. Summed nerve activity during turning movements. Activity data from the nerve recordings shown in Figure 15 were summed and are plotted as percent of baseline activity for ipsilatcral $(A)$ and contralateral $(B)$ turning movements (averaged per 1 -sec time interval to allow comparison with the data obtained from the calculations). Note that there is a substantial increase in unit activity during the first $2 \mathrm{sec}$ of the turning movement in the ipsilateral direction (duration of the turning movement is indicated by the bar). During the turning movement in the contralateral direction, the nerve activity is not reduced, and an increase in activity occurred at the termination of the movement.

the contralateral turning movements. The summed activity strongly increased only during the first $2 \mathrm{sec}$ of the turning movement in the ipsilateral direction (Fig. 16A). Contralateral turning movements (Fig. 16B) were not associated with a reduction in overall unit activity. However, an increase in unit activity correlated with the termination of the turning movements, perhaps reflecting activity associated with braking of the movements.

\section{Discussion}

Our analysis of head movements of Aplysia in response to food stimulation at different loci revealed a variety of fundamentally different responses: defensive withdrawal of the head or appetitive head lifting, which are both relatively undirected responses. Furthermore, once the animal is oriented in the headup position, the same stimulus leads to a directed turning movement in the direction of the stimulus. The schematic diagram of these directed turning movements is shown in Figure 17 , which illustrates that the position of the stimulus determines the magnitude and direction of the responses (represented by the vectors pointing in a quasi 3-dimensional space). The current findings demonstrate that the eccentricity of the stimulus determines the magnitude of the turning movements. This rule applies to stimuli near the mouth, which evoke movements in the horizontal plane. More eccentric stimuli (e.g., on the tips of the tentacles) add a vertical component to the horizontal turning movement. Stimulation on the back of the head causes the animal to arch almost vertically downwards, as indicated by the vertically pointing arrow in Figure 17. The strongest downwarddirected movement, however, was not evoked by stimulating the rhinophores, which were the most eccentric part tested (mea-

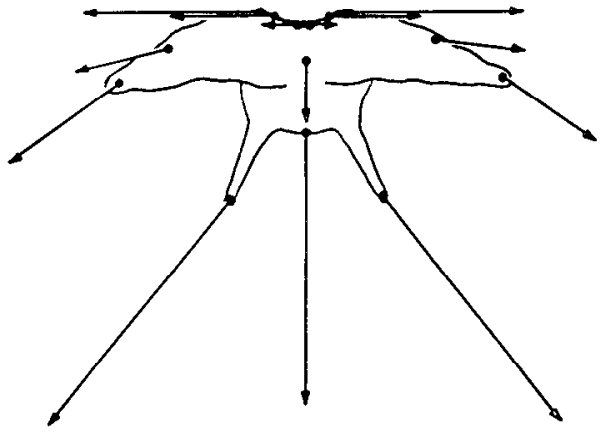

Figure 17. Schematic representation of direction and magnitude of turning responses of animal when stimulated at different locations on head. Averaged data of 2 responses for each stimulus position are shown (front view; ventral surface is up). A horizontal turning response is indicated by a horizontal vector; a downward arching, by a vector in the vertical direction. The stimulus position is indicated by the $(d o t)$ at the origins of the vectors.

sured with respect to the mouth), but by the neck portion anterior to the rhinophores. Hence, the magnitude of the response increases gradually to a maximum at a certain eccentricity, then declines for further eccentric loci.

A striking feature of the turning response of the animal under open-loop conditions (brief stimulation), is that though the response is a function of the stimulus position, the response results in a large overshoot of the mouth beyond the target (stimulus) position. We demonstrated that the overshoot is not an artifact due to chemical residues of the seaweed stimulus remaining on the receptive surface, because a very similar response can be evoked by a tactile stimulus on the same spot. This suggests that the turning response of the animal to a transient stimulus, serves to rapidly turn the animal in the direction of the stimulus, rather than to directly locate the food. It is possible that overshooting responses are advantageous in the natural habitat when it might be important for the animal to turn quickly in the direction of a seaweed frond that contacts the animal briefly, but is moved to the side by water currents. The overshooting open-loop response of Aplysia contrasts with the several relatively accurate triggered orienting responses of other invertebrates. For example, open-loop head movements in mantids are extremely accurate (Lea and Mueller, 1977), as are the tactually evoked attack responses of crayfish (Zeil et al., 1985), though additional sensory feedback increases the accuracy of the movements. It is interesting that turning responses in crayfish, in contrast to those in Aplysia, tend to undershoot the target position in the absence of sensory feedback (Copp and Watson, 1988). Furthermore, in vertebrates, the body orientation to tactile stimuli in frogs (Comer and Grobstein, 1981) and saccadic eye movements in mammals (for review, see Robinson, 1981) are examples of extremely accurate triggered responses.

Under closed-loop conditions, that is, when the animal is able to maintain lip contact with the stimulus, it can quite accurately orient its mouth to the food. Thus, the precise turning movements of Aplysia have to be stirnulus guided rather than stimulus triggered. This raises the question as to how the overshooting character of the turning response under open-loop conditions is transformed into the more accurate orienting response that characterizes the closed-loop situation, because under both conditions, the stimulus is initially applied at the same spot on the lip. The data presented in this paper suggest that the higher 
accuracy of the response under closed-loop conditions cannot be accounted for either by the prolonged presence of the stimulus under closed-loop conditions or by adjustments made after the stimulus contacts the opposite side of the mouth. A possible braking effect of the biting program on the turning response also does not appear to explain the results. The experiments suggest that the critical factor essential for accurate orientation is the movement of the stimulus along the receptive surface. As the stimulus slides from lateral to more medial loci, the larger response that was triggered laterally is substituted by progressively smaller responses, which are characteristic for more medial stimulation. This kind of response substitution mechanism would convert stronger turning responses into weaker responses when the stimulus contacts more medial areas. Under closed-loop conditions, when the stimulus moves closer and closer to the mouth, previously triggered stronger responses will be substituted for by subsequently evoked weaker responses, and the head will ultimately stop turning when the food is located directly at the mouth. If the animal nevertheless misses the food, that is, if it moves its mouth past the food so that the opposite side of the mouth now contacts the food, the same mechanism will lead the animal back in the direction of the food. Support for the notion of response substitution was provided by experiments in which a turning response was triggered by lateral stimulation, and while the animal was turning, a second test stimulus was delivered on the same side but at a more medial location. The resulting turning movement started in a way typical for lateral stimulation, but following the second stimulus, the movement continued at a reduced velocity and terminated at a significantly reduced angle. The angle turned following the second (medial) stimulus corresponds approximately to the net turning angle expected for the medial stimulus alone.

A stimulus delivered while the animal exhibits spontaneous or evoked biting is not effective in evoking a turning response, though the occurrence of bites does not interfere with ongoing turning movements. Therefore, the most likely explanation as to why stimulation during biting fails to evoke a turning response is that some aspects of the biting program gate out the sensory input that triggers the turning response. This gating mechanism makes sense functionally, because it would be detrimental for the animal to turn toward a new piece of food while feeding and therefore possibly lose its food. An inhibitory influence of the feeding system on other behaviors has been described earlier in Aplysia (Advokat, 1980) and in Pleurobranchaea, where it has been discussed in the context of behavioral hierarchy (Kovacs and Davis, 1980). In Pleurobranchaea, as in Aplysia, withdrawal responses are suppressed during feeding. In Pleurbranchaea, for the suppression of withdrawal, feeding movements have to occur (Davis et al., 1977), but the source of the inhibition, which could lie either in the bite command or in the actual buccal motor program, has not been identified. Data obtained in Aplysia with lesions of the C-B connectives provide evidence that the gating signal influencing the turning movements is generated in the cerebral ganglion and does not require a buccal molor program. This suggests that the gating signal originates in higherorder command elements located in the cerebral ganglion. A command element for the biting program has been identified by Rosen et al. (1988) in the cerebral ganglion, and further studies should allow a test of the hypothesis that a gating signal originates in the command elements for biting. We have found that the inhibition is most pronounced during the initial phases of the biting program, namely, lip closing and protraction of the radula, and is not present during retraction. In Lymnaea, which feed while locomoting, inhibitory input to pedal motor neurons occurs during the retraction phase of the radula cycle (Kyriakides and McCrohan, 1988). The inhibition is abolished by cutting the C-B connectives, indicating that the inhibitory input in this species comes from the buccal ganglion. However, it should be kept in mind that the feeding behavior of Lymnaea and Aplysia differ in many respects, and therefore, the inhibition associated with biting may not be related.

Food-aroused animals turn in a similar way to purely tactile stimuli as to food (see also Preston and Lee, 1973). On repeated tactile stimulation, however, the magnitude of the turning response decrements, and the animals eventually stop responding. A decrement of consummatory (biting) responses to pure tactile stimuli has been described for Aplysia vaccaria (Lickey, 1968). This addresses the question of the specific role of the chemical component in a stimulus, such as seaweed, which provides both chemical and tactile components. By stimulating the animals selectively with either a purely tactile or a purely chemical stimulus, we found that chemical stimulation is ineffective in evoking turning responses. Tactile stimulation, on the other hand, reliably evoked turning and biting responses, but only if the animal was intermittently stimulated with a chemical stimulus (see also Rosen et al., 1982). This indicates that the chemical component of seaweed appears to contribute very little (or not at all) to the turning response, but more importantly, chemical stimuli are critical for maintaining the food-arousal state of the animal. As a consequence, the compound of tactile and chemical components in seaweed can effectively trigger turning responses of a steady magnitude. We have recently identified a putative command element for the food-arousal statc in Aplysia (Teyke et al., 1990), which responds vigorously to seeweed stimulation of the tentacle and rhinophores, whereas tactile stimulation evokes a significantly weaker response (T. Teyke, unpublished observations).

To obtain information about the possible neural basis of the turning responses, the torque necessary to generate the turning movements was calculated by using a physical model. On the basis of this simulation, we predicted that the turning movements have to be powered for only about half the duration of the actual movement. The simulation further indicated the presence of a counter-directed braking force that terminates the movements. We obtained support for both predictions by extracellular nerve recordings, which showed that, in the majority of cases, a strong increase in unit activity occurred within the first $2 \mathrm{sec}$ of ipsilateral turning movements. During contralateral turning movements, unit activity increased mostly at the termination of the movement in different pedal nerves that innervate different parts of the neck region. This can indicate that, in order to brake the turning movements, the animals adjust their body tonus by a concerted action of several nerves and several muscle groups, rather than by generating a strictly counter-directed braking force. The involvement of several nerves in head-turning movements is further supported by the finding that animals with lesions of a single neck nerve perform turning movements without any detectable deficits ( $T$. Teyke, unpublished observations).

Based on the present experiments, it is not possible to present a detailed neural model of directed turning in Aplysia. However, in Figure 18, we present a heuristic scheme of the key elements that can be postulated based on our current results. We suggest that the directed turning response is due to a projection of the 


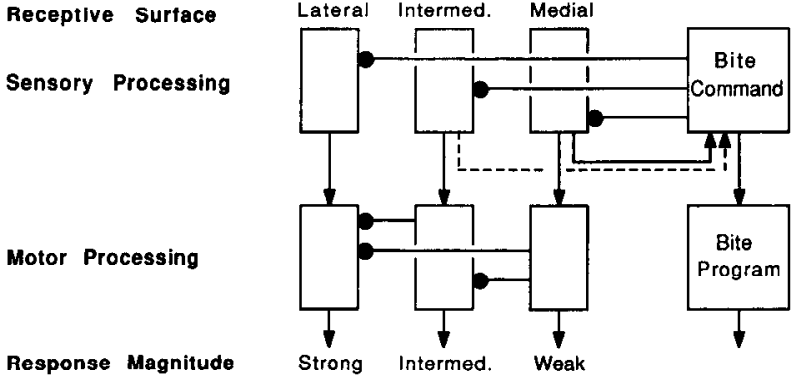

Figure 18. Schematic block diagram of postulated neuronal mechanisms underlying directed turning movements of Aplysia. Functional connections are drawn (dots, inhibitory; arrows, excitatory). The dotted line indicates a weak connection.

receptive surfaces of the lip and tentacle onto discrete motor elements. Sensory stimulation is postulated to evoke relatively prolonged responses in sensory processing elements. More lateral stimuli evoke progresively stronger responses. Inhibitory connections from medial to lateral motor processing modules account for the finding that medial stimulation suppresses the responses evoked by more lateral stimuli, thus substituting weaker for stronger responses. The model also shows that medial stimulation and, to a lesser extent (Fig. 18, dashed line), intermediate stimulation excite a command system for biting. Our experiments further indicate that the bite command provides the signal that gates out the capacity of stimuli to evoke a turn, and consequently, the model shows input from the bite command providing inhibition at the level of the sensory processing units. The postulated neural system does not function unless the animal is first aroused by chemical stimuli provided by food. The arousal effects could be mediated by the C-PR neuron, which has been shown to be excited by seaweed stimulation of the lips of the animal and to evoke neural activity that can control appetitive behaviors such as head lifting (Teyke et al., 1990). The site of action of the arousal effects is not shown in the model, because the current data provide no indication of the mechanism, and the arousal effects could operate at 1 or more levels. Although the model lacks details of the interconnections between units, it nevertheless provides the broad outlines of testable ideas.

\section{References}

Advokat C (1980) Modulation of defensive reflexes in Aplysia californica by appetitive stimulation. Behav Neural Biol 28:253-265.

Bablanian GM, Weiss KR, Kupfermann I (1987) Motor control of the appetitive phase of feeding behavior in Aplysia. Behav Neural Biol 48:394-407.

Barmack NH (1976) Measurements of stiffness of extra ocular muscles of the rabbit. J Neurophysiol 39:1009-1019.

Bizzi E, Dev P, Morasso P, Polit A (1978) Effect of load disturbances during centrally initiated movements. J Neurophysiol 41:542-556.

Comer C, Grobstein P (1981) Tactile elicited prey acquisition behavior in the frog, Rana pipiens, and a comparison with visually elicited behavior. J Comp Physiol 142:141-150.
Cook DG, Carew TJ (1989) Operant conditioning of head-waving in Aplysia. III. Cellular analysis of possible reinforcement pathways. J Neurosci 9:3115-3122.

Copp NH, Watson D (1988) Visual control of turning responses to tactile stimuli in the crayfish Procambarus clarkii. J Comp Physiol 163:175-186.

Craig W (1918) Appetites and aversions as constituents of instincts. Biol Bull 34:91-107.

Cropper EC, Kupfermann I, Weiss KR (1990) Differential firing patterns of the peptide-containing cholinergic motor neurons B15 and B16 during feeding behavior in Aplysia. Brain Res 522:176-179.

Davis WJ, Mpitsos GJ, Pinneo JM, Ram JL (1977) Modification of the behavioral hierarchy of Pleurobranchaea. I. Satiation and feeding motivation. J Comp Physiol 117:99-125.

Frings H, Frings C (1965) Chemosensory bases of food-finding and feeding in Aplysia juliana (Mollusca, Opisthobranchia). Biol Bull 128: 211-217.

Kovacs MP, Davis WJ (1980) Reciprocal inhibition between feeding and withdrawal behaviors in Pleurobranchaea. J Comp Physiol 139: $77-86$.

Kupfermann I (1974a) Feeding behavior in Aplysia: a simple system for the study of motivation. Behav Biol 10:1-26.

Kupfermann I (1974b) Dissociation of appetitive and consummatory phases of feeding behavior in Aplysia: a lesion study. Behav Biol 10: 89-97.

Kyriakides MA, McCrohan CR (1988) Central coordination of buccal and pedal neuronal activity in the pond snail Lymnaea stagnalis. $\mathbf{J}$ Exp Biol 136:103-123.

Lea JY, Mueller CG (1977) Saccadic head movements in mantids. J Comp Physiol 114:115-128.

Lickey ME (1968) Learned behavior in Aplysia vaccaria. J Comp Physiol Psychol 66:712-718.

Preston RJ, Lee RM (1973) Feeding behavior in Aplysia californica. Role of chemical and tactile stimuli. J Comp Physiol Psychol 82:368381.

Robinson DA (1981) Control of eye movements. In: Handbook of physiology, Sec 1, The nervous system, Vol II, Pt 2 (Brookhart JM, Mountcastle VB, eds), pp 1275-1320. Bethesda: American Physiological Society.

Rosen SC, Wciss KR, Kupfermann I (1982) Cross-modality sensory integration in the control of feeding in Aplysia. Behav Neural Biol 35:56-63.

Rosen SC, Miller MW, Weiss KR, Kupfermann I (1988) Activity of CBl-2 of Aplysia elicits biting-like responses. Soc Neurosci Abstr 14: 608.

Stark L, Zangemeister WH, Harnford B (1988) Head movement models, optimal control theory, and clinical applications. In: Control of head movements (Peterson BW, Richmond FJ, eds), pp 245-260. New York: Oxford UP.

Susswein AJ, Weiss KR, Kupfermann I (1978) The effects of food arousal on the latency of biting in Aplysia. J Comp Physiol 123:3141.

Teyke T, Weiss KR, Kupfermann I (1990) An identified neuron (CPR) cvokes ncuronal responses reflccting food arousal in Aplysia. Science $247: 85-87$.

Weiss KR, Cohen J, Kupfermann I (1978) Modulatory control of buccal musculature by a serotonergic neuron (metacerebral cell) in Aplysia. J Neurophysiol 41:181-203.

Winer BJ (1971) Statistical principles in experimental design. New York: McGraw-Hill.

Zeil J, Sandeman R, Sandeman D (1985) Tactile localization: the function of active antennal movements in the crayfish Cherax destructor. J Comp Physiol 157:607-617. 